\title{
Rho GTPase-activating protein 1 promotes apoptosis of myocardial cells in an ischemic cardiomyopathy model
}

\author{
Xuezhi Zhang ${ }^{1}$, Jun Guan², Minglei Guo ${ }^{1}$, Hongyan Dai², Shanglang Cai ${ }^{3}$, Changyong Zhou'1, Yongbin Wang ${ }^{1}$, Qiaoji Qin' \\ 1 Department of Emergency Internal Medicine, Affiliated Hospital of Qingdao University, Qingdao, China \\ 2 Department of Cardiology, Qingdao Municipal Hospital, Qingdao, China \\ 3 Department of Cardiology, Affiliated Hospital of Qingdao University, Qingdao, China
}

\section{KEY WORDS}

apoptosis, ARHGAP1, hypoxia, ischemic cardiomyopathy, myocardial cells
Correspondence to: Prof. Qiaoji Qin, No. 16 Jiangsu Road, Qingdao, Shandong Province, 266003 , China, phone: +86053282911201, email: hi1519@sina.com Received: October 17, 2019. Accepted: October 25, 2019. Published online: October 30, 2019. Kardiol Pol. 2019; 77 (12): 1163-1169 doi:10.33963/KP.15040 Copyright by the Author(s), 2019

\begin{abstract}
BACKGROUND Ischemic cardiomyopathy (ICM) leads to heart failure by causing apoptosis of cardiac myocytes. It is generally believed that a therapy targeting apoptosis of cardiac myocytes would improve the prognosis of patients with ischemic heart disease.

AIMS We aimed to investigate the role of Rho GTPase-activating protein 1 (ARHGAP1) in ICM.

METHODS The cellular model of myocardial ischemia ( $\mathrm{H} 9 \mathrm{c} 2$ cell model) and a rat model of ICM were established to explore the expression of ARHGAP1. The overexpression of ARHGAP1 was induced in H9c2 myocardial cells to assess protein function.

RESULTS The expression of ARHGAP1 as a result of hypoxic conditions in the cellular and rat models was observed. Its overexpression induced apoptosis of cultured $\mathrm{H} 9 \mathrm{c} 2$ cells under normal atmospheric conditions. ARHGAP1 was also shown to initiate the apoptosis pathway by regulating the cell death modulators B-cell lymphoma 2 (BCl-2) and BCl-2-associated X protein.

CONCLUSIONS Our results show that the ARHGAP1 expression is closely associated with apoptosis of myocardial cells, which in turn leads to ICM. Thus, ARHGAP1 may become a novel molecular marker of the hypoxia-induced apoptosis pathway and serve as a potential therapeutic target in patients with ICM.
\end{abstract}

INTRODUCTION Ischemic cardiomyopathy (ICM) is the most common type of heart disease, with a high rate of sudden cardiac death. The direct cause of ICM is usually insufficient blood and oxygen supply to the heart muscle, ${ }^{1}$ which leads to sustained cardiac cell death, cardiac dysfunction, and ultimately heart function deterioration. Coronary artery stenosis, myocyte death, reactive cellular hypertrophy, and ventricular scarring are all associated with heart failure. Among the numerous complex molecular mechanisms underlying ICM, the role of apoptosis and antiapoptotic therapies has attracted a considerable attention. ${ }^{2}$ Uncovering the underlying mechanism and the effectors of apoptosis would help identify a clear signaling pathway of heart failure. This, in turn, would enable the development of a potential therapeutic target, or at least a pathological marker that could aid in the diagnosis of heart diseases.

By searching 4 databases associated with human ICM samples in the Gene Expression Omnibus database, a total of 26 differentially expressed genes (ASPN, PHLDA1, PENK, TSPAN9, ILF3, SEC14L1, SVIL, PLCE1, CCNG2, ARHGAP1, FCN3, MYH6, HOPX, SERPINA3, PLA2G2A, LYVE1, S100A9, ENO1, PTP4A2, TM4SF1, CDK2, ATG101, SLC1A1, P2RY2, AGFG1, and FLII) were identified. ${ }^{3-5}$ Compared with normal myocardial tissue, ARHGAP1 had a significantly upregulated expression in ICM.

The specific function of ARHGAP1 is poorly understood, but there is considerable evidence to show that it plays a critical role in gene expression 


\section{WHAT'S NEW?}

In this research, the involvement of Rho GTPase-activating protein 1 (ARHGAP1) in hypoxia-induced apoptosis was elucidated for the first time. The role of this association in the hypoxia-induced apoptosis pathway was explored. As a potentially reversible cause of cardiac function deterioration, ARHGAP1 may become a novel molecular marker and therapy target in ischemic cardiomyopathy.

regulation as well as cell cycle, migration, and apoptosis..$^{6-9}$ ARHGAP1 was reported to interact with $\mathrm{p} 53$, resulting in cell-cycle arrest and apoptosis, ${ }^{10}$ and it was also shown to regulate c-Jun N-terminal kinase-mediated apoptosis in vivo. ${ }^{11,12}$

There have been few studies reporting on the role of ARHGAP1 in heart diseases, particularly in ICM. Therefore, we decided to investigate a potential correlation between ARHGAP1 and apoptosis. Using a cellular model of myocardial ischemia and a rat model of ICM, we assessed ARHGAP1 overexpression with real-time quantitative polymerase chain reaction (qPCR), Western blotting, and immunohistochemical staining. To further analyze its role, we induced gene overexpression in cardiomyocytes. This directly limited cell growth and initiated apoptosis by downregulating growth effectors and activating cell death modulators.

METHODS Cell culture and transfection Rat cardiac cells, H9c2 (American Type Culture Collection, Rockville, Maryland, United States), were cultured in DMEM medium (Gibco, Grand Island, New York, United States), supplemented with $10 \%$ fetal bovine serum (FBS) in a humidified atmosphere of $5 \% \mathrm{CO}_{2}$ at $37^{\circ} \mathrm{C}$.

To stimulate hypoxia, high-glucose DMEM medium with $10 \%$ FBS was substituted with non-glucose DMEM to imitate ischemia. Then, H9c2 cells were incubated at $37^{\circ} \mathrm{C}$ in an atmosphere of $94 \% \mathrm{~N}_{2}, 5 \% \mathrm{CO}_{2}$, and $1 \% \mathrm{O}_{2}$, instead of $95 \%$ air and $5 \% \mathrm{CO}_{2}$, for 24 hours.

Transfection was performed with Lipofectamine 2000 (Invitrogen, Grand Island, New York, United States) according to the manufacturer's instruction. Cells were harvested for qPCR or Western blot 48 hours posttransfection. The sequence of small interfering RNA (siRNA) is 5'-ACUAAACACAAUGAUCUUCCG-3', and the negative control sequence of siRNA is 5'-GCTGGTTGATCGAGTAGATTCTA-3'.

Cloning construction The ARHGAP1 gene was synthesized from Genewiz (South Plainfield, New Jersey, United States), digested with EcoRI and HindIII enzymes, and then ligated into the vector of pcDNA3.1. After sequencing verification, the ARHGAP1 overexpression vector was obtained. Cloning primers were ARHGAP1-xho-F: agaCTCGAGatggatccgctctcagagc and ARHGAP1-Hind-R: tttAAGCTTtcagagcccgctggggtcc.
Quantitative polymerase chain reaction assay Total RNA was extracted from the cultured cells or model rat tissues using TRIzol Reagent (Invitrogen) according to the protocol. Extracted RNAs were transcribed into complementary DNA (cDNA) using First Strand cDNA Synthesis Kit (Marligen Bioscience, Ijamsville, Maryland, United States) and used for template. The qPCR amplification was performed with SYBR Premix Ex Taq II (Takara Biotechnology, Dalian, Liaoning, China), and primers were designed and provided by GeneCopoeia (Hongxun, Suzhou, Jiangsu, China). The relative quantitative values were calculated using the comparative threshold cycle $\left(2^{-\triangle \Delta C T}\right)$ method. The qPCR for each cDNA sample was carried out in triplicate.

Western blot analysis Cells were homogenized and lysed in pre-cold radio-immunoprecipitation assay lysis buffer plus protease inhibitors (Roche Diagnostics, Indianapolis, Indiana, United States). An equal amount of protein samples was loaded and separated in $10 \%$ SDS-PAGE, then transferred to a polyvinylidene fluoride membrane (BD Pharmingen Inc., San Diego, California, United States). The membranes were blocked by incubation with $5 \%$ fat-free milk in tris-buffered saline $(\mathrm{NaCl}$, 150 mM; Tris- $\mathrm{HCl}, 50$ mM; Tween-20, 0.5\%l; $\mathrm{pH}, 7.6)$ for 1 hour, incubated with primary antibodies for 1 hour, and then incubated with horseradish peroxidase-conjugated secondary antibody for 1 hour. Finally, the blots were developed with an enhanced chemiluminescence reagent (Thermo Fisher Scientific, Rockford, Illinois, United States). All reactions were developed at room temperature. The following antibodies were used for immunoblotting: mouse monoclonal anti- $\beta$-tubulin antibodies, rabbit polyclonal anti-Bcl-2 antibodies, as well as rabbit polyclonal anti-ARHGAP1, anti-Bax, anti-caspase-3, anti-caspase-3 (cleaved Asp175), antimatrix metallopeptidase-2 (MMP2), anti-cyclin B1 (CCNB1), and anti-proliferating cell nuclear antigen (PCNA) antibodies (GeneTex, Inc., Irvine, California, United States). The expression of $\beta$-tubulin was set as control.

Immunohistochemistry Rat cardiac muscle tissue samples were fixed with paraformaldehyde and cut into 4- $\mu \mathrm{m}$ sections. Anti-ARHGAP1 antibodies were applied to the sections and incubated overnight at a temperature of $4^{\circ} \mathrm{C}$. After incubation with secondary antibodies conjugated with streptavidin-horseradish peroxidase for 1 hour, the expression and distribution of ARHGAP1 were visualized using 3,3'-diaminobenzidine. All sections with positive staining were assessed microscopically.

Cell proliferation assay The H9c2 cells transfected with siRNA or plasmid were cultured 
in a 96-well plate for 24, 48, and 72 hours at $37^{\circ} \mathrm{C}$. Subsequently, the growth rates were determined using the Cell Counting Kit- 8 assay by analyzing the absorbance at a wavelength of $450 \mathrm{~nm}$ using the VersaMax Microplate Reader (Molecular Devices, Sunnyvale, California, United States). All experiments were performed in triplicate.

Migration and invasion assay Cell migration was performed in a pre-made dual chamber containing a polycarbonate membrane with an 8 - $\mu$ m pore diameter. Cells in serum-free medium were seeded on the upper compartment, and complete medium was added to the lower compartment. After incubation at a temperature of $37^{\circ} \mathrm{C}$ for 1 hour and fixing with $4 \%$ paraformaldehyde, nontraversed cells were washed from the upper surface of the filter, while traversed cells were stained with crystal violet and counted on the lower side of the filter. The method used for cell invasion was the same as for cell migration except that the membranes had to be precoated with matrigel (BD Biosciences, San Jose, California, United States).

Apoptosis assay Flow cytometry was used to investigate apoptosis by Annexin V-FITC/PI Staining / Detection Kit (Abcam, Shanghai, China). After transfecting with siRNA or plasmid, the H9c2 cells were harvested 48 hours later and washed with precooling phosphate-buffered saline, then resuspended to $2 \times 10^{5}$ cells $/ \mathrm{ml}$. Then, $5 \mu \mathrm{l}$ of fluorochrome-conjugated annexin $\mathrm{V}$ was added to $100 \mu \mathrm{l}$ of cells and incubated at room temperature for 10 minutes. After washing, $5 \mu \mathrm{l}$ of fresh propidium iodide solution was added and incubated at $37^{\circ} \mathrm{C}$ for 30 minutes in the dark. Finally, cell apoptosis was assessed using the C6 flow cytometer (BD Biosciences).

Lactate dehydrogenase release assay Lactate dehydrogenase (LDH) activity was determined by LDH Cytotoxicity Assay Kit (\#88953, Thermo Fisher Scientific), according to the manufacturer's instruction.

Rat model of ischemic cardiomyopathy Myocardial infarction was performed by surgical ligation of the left anterior descending coronary artery. Healthy male Sprague-Dawley rats, weighing between $220 \mathrm{~g}$ and $250 \mathrm{~g}$, were used. Rats were anesthetized by an intraperitoneal injection of sodium pentobarbital (30 mg/kg). The chest was opened at the left fourth intercostal space to expose the heart, and the left anterior descending coronary artery was ligated by a $6-0$ silk suture $1 \mathrm{~mm}$ below the tip of the left atrial appendage. Successful ligation was verified by a color change. For the control groups, the left chest was opened without any invasive procedure of the coronary artery.
Eight rats were treated following the surgery for each group.

Ethics statement All animals were treated in accordance with the Guide for the Care and Use of Laboratory Animals. All experiments were approved by the Ethics Committee of the Affiliated Hospital of Qingdao University, Shandong, China (approval number: 2017030601), and performed according to its guidelines.

Statistical analysis Data were presented as mean (SD). Statistical analysis was performed using the $t$ test or 1-way analysis of variance, using the GraphPad Prism 6.0 software (GraphPad Software Inc., San Diego, California, United States). All experiments were performed at least in triplicate. A $P$ value of less than 0.05 was considered significant.

RESULTS Association of cell migration and proliferation with ARHGAP1 in a cellular model of myocardial ischemia To simulate ischemia, H9c2 myocardial cells were cultured under hypoxic conditions for 24 hours. Then, the growth parameters of the cultured cells were compared with those of the control group (cells grown under normal conditions). Through cell staining, we found that the migration and invasion abilities of H9c2 cells grown under hypoxic conditions were reduced (FIGURE $1 A$ and Supplementary material, Figure S1A). The number of migration cells decreased to approximately $30 \%$ of the control group (Supplementary material, Figure $S 1 B$ and $S 1 C$ ). The results of the Cell Counting Kit- 8 assay revealed that the cell viability reduced along with an increase in hypoxia duration (FIGURE $1 B$ ). The viability of H9c2 cells cultured under hypoxic conditions for 72 hours dropped to $70 \%$ of that observed for control cells. Simulation of the ischemic conditions induced lactate accumulation in the H9c2 cells, which is an indicator of tissue damage under hypoxic conditions. The levels of LDH in the test cells were up to 3.5-fold higher than the levels in the control group (Supplementary material, Figure S1D). Our results further confirmed hypoxic damage based on the release of LDH.

It has been reported that hypoxia itself cannot induce apoptosis, which requires the involvement of the frequently activated caspase protein, caspase- 3 . $^{2} \mathrm{Bcl}-2$ is an important protein family that mediates the apoptotic response in cells..$^{13,14}$ This protein family contains 2 groups of structurally-related proteins: antiapoptotic and proapoptotic. The functional balance between these 2 groups influences the fate of the cells. The Bcl-2 protein has an antiapoptotic effect, whereas the Bax protein has an apoptotic effect. ${ }^{15}$ Both proteins regulate the release of cytochrome $\mathrm{c}$ and the activation 


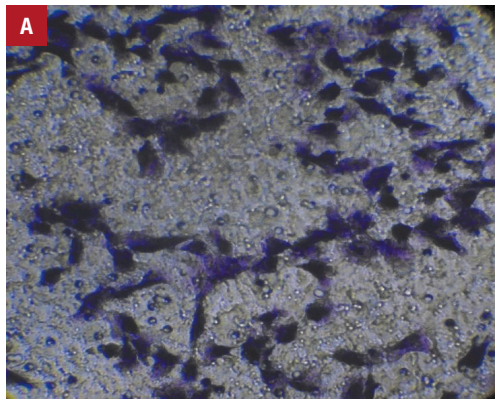

Control

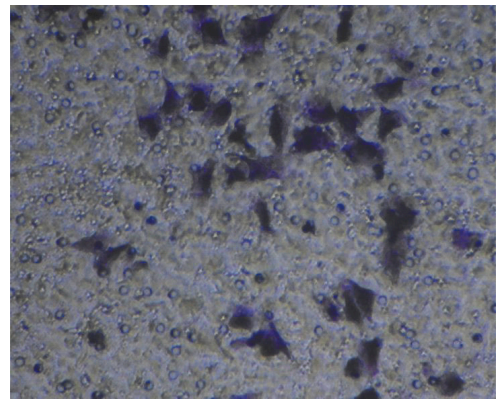

Hypoxia

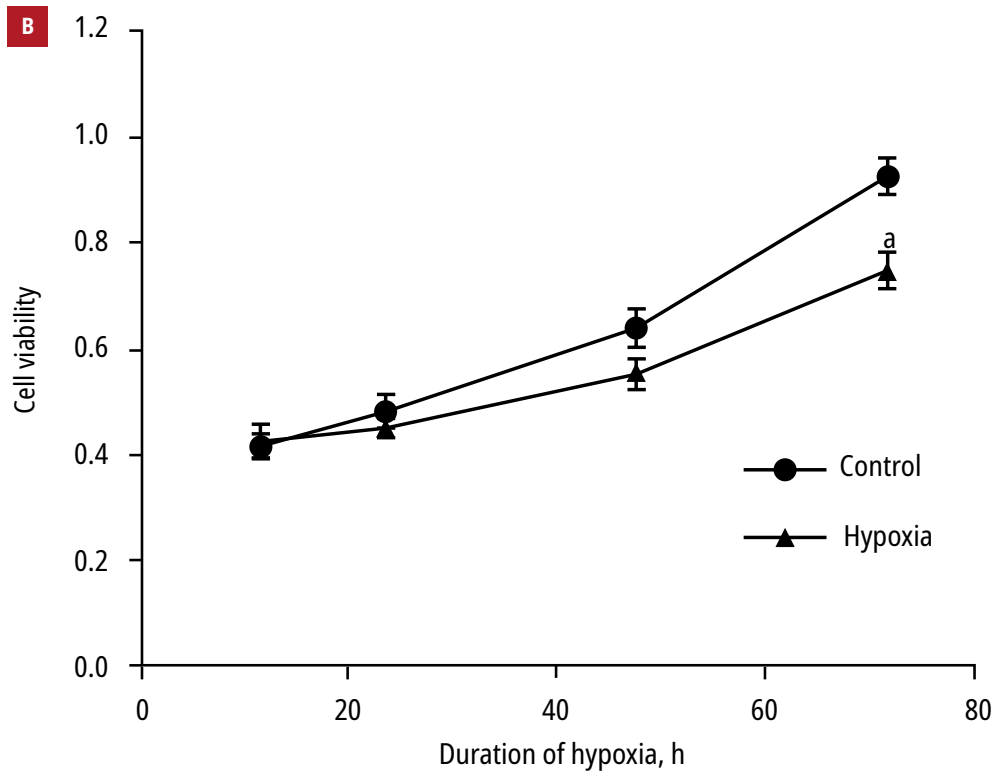

FIGURE 1 Cell proliferation in the cellular model of myocardial ischemia: A - migration of H9c2 cells was weakened under hypoxic conditions compared with normal atmosphere (control group; magnification $\times 200$ ); B - viability of H9c2 cells under hypoxic conditions was time dependent, and it decreased from 12 hours to 72 hours. All data are presented as mean (SD) activity from at least 3 independent experiments $(n>3)$ for each condition; a $P<0.01$ vs control
A

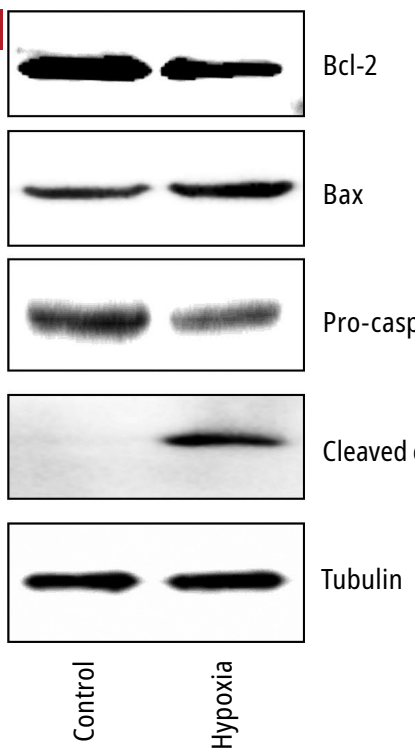

B
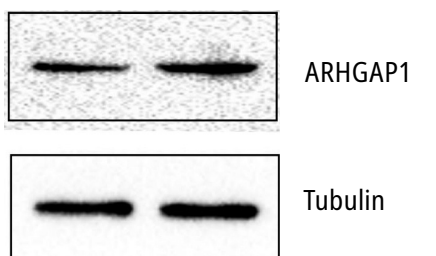

FIGURE 2 Expression of apoptosis modulators and ARHGAP1 in H9c2 cells detected by Western blot: A - BCl-2, Bax, pro-caspase, and cleaved caspase-3 expression in H9c2 cells cultured under hypoxic conditions; B - ARHGAP1 expression in control and hypoxic cells of caspase-3. ${ }^{14,16}$ In our study, chronic hypoxia induced the expression of Bax and inhibited the expression of Bcl-2, while simultaneously activating caspase- 3 . These results were quantified and verified by Western blot (FIGURE2A; Supplementary material, Figure S2A).

Under the same hypoxic conditions, ARHGAP1 overexpression was detected by qPCR and Western blot. The ARHGAP1 mRNA expression doubled compared with that in the control cells at 24 hours posthypoxia (Supplementary material, Figure S2B). The levels of the ARHGAP1 protein were shown to increase by 4 -fold, as assessed by Western blot (FIGURE 2B; Supplementary material, Figure S2C). All these results suggested a significant correlation between ARHGAP1 and ICM.

ARHGAP1 expression in a rat model of ischemic cardiomyopathy The ICM rat model was established using the coronary artery ligation method to induce myocardial infarction. The expression and distribution of ARHGAP1 were analyzed 28 days after surgery by immunohistochemical staining and Western blot. Staining revealed that ARHGAP1 levels were significantly increased in test samples, as compared with those in control samples (FIGURE 3A). Statistical analysis based on Western blot revealed that the mean level of ARHGAP1 in test samples was 2-fold higher than that in control samples (Supplementary material, Figure S3). Four samples from the ICM rat model and control group were displayed randomly (FIGURE 3B). Briefly, the ARHGAP1 expression was elevated in the ICM rat model, which was consistent with the results observed for $\mathrm{H} 9 \mathrm{c} 2$ cells.

Effect of ARHGAP1 overexpression on cell proliferation and apoptosis ARHGAP1 has been reported to be involved in cell apoptosis in embryonic fibroblasts and other organs. ${ }^{12}$ However, there is no evidence that would clarify its function in the heart. In our study, protein levels in the H9c2 cell model, as well as the ICM rat model, implied that ARHGAP1 is overexpressed under hypoxic conditions. To explore its role in ICM, we developed an expression vector to induce ARHGAP1 overexpression in H9c2 cells. As a comparison, the endogenous ARHGAP1 gene was knocked down mediated by siRNA as a control. Blank vectors and unrelated siRNA were transfected into H9c2 cells to set as ARHGAP1 overexpression and knockdown control separately. The resulting cell line was analyzed, and the apoptosis rates were quantified by the Annexin V-FITC/PI Staining / Detection Kit, based on flow cytometry methods. Compared with the blank control group (untreated cells), cells transfected with the overexpression vector (ARHGAP1+) showed a $13.3 \%$ higher expression of ARHGAP1. The ratios of the cells transfected 


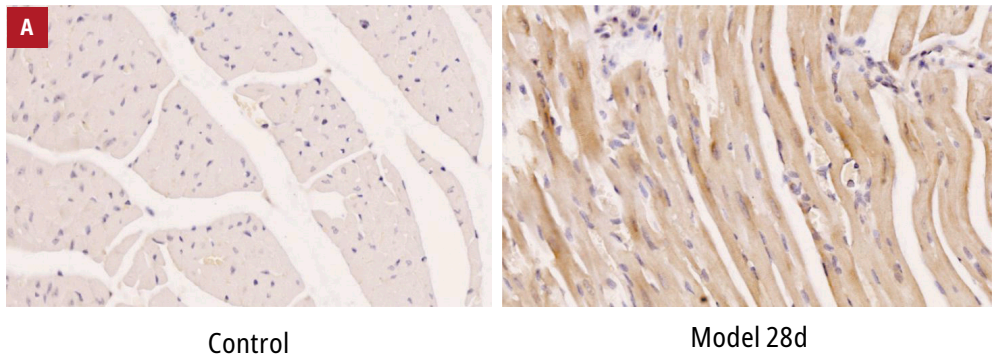

B

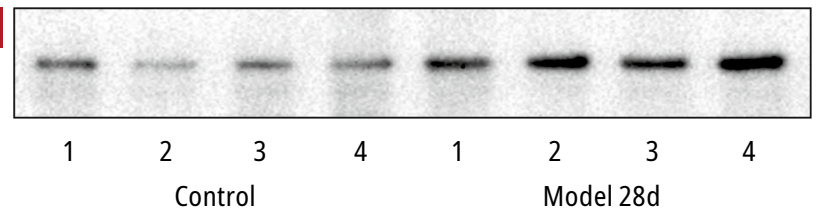

FIGURE 3 Increased ARHGAP1 expression in the rat model of ischemic cardiomyopathy (ICM); the ventricular tissues from the control rats (left) and ICM rats (right) after 28 days of coronary artery ligation were subjected to immunohistochemical staining (A) and Western blot (B). Western blot results indicated ARHGAP1 overexpression. Each group included 8 rats, and 4 samples of each were randomly displayed.
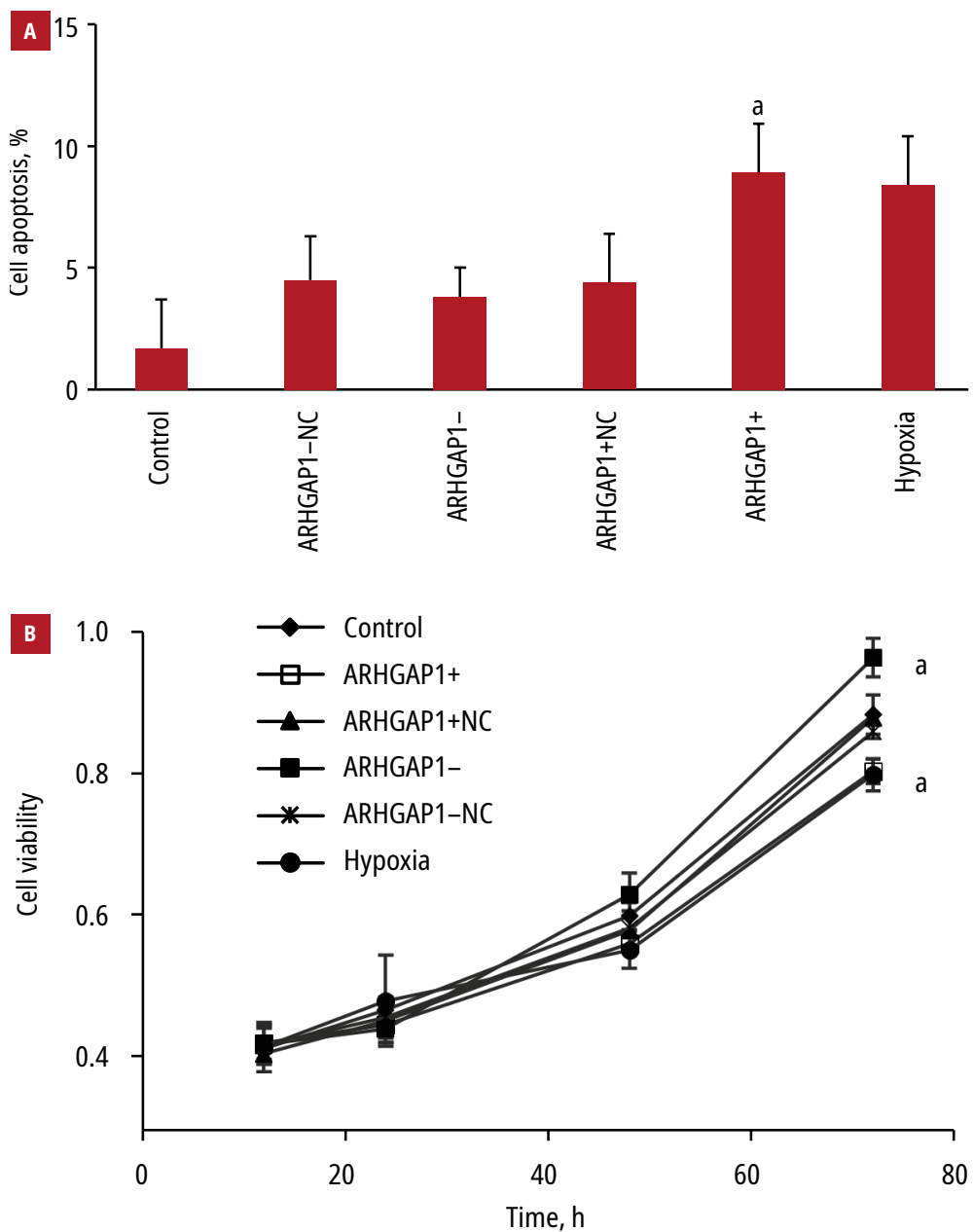

FIGURE 4 Effect of ARHGAP1 overexpression on cell apoptosis and survival. There were 6 groups: 1) control, untreated H9c2 cells; 2) ARHGAP1-, cells transfected with siRNA targeting ARHGAP1;3) ARHGAP1-NC, cells transfected with irrelevant siRNA sequence; 4) ARHGAP1+, cells containing vectors overexpressing ARHGAP1; 5) ARHGAP1+NC, cells with only blank vectors; and 6) positive control, cells cultured under hypoxic conditions. A - the cell apoptosis ratio based on flow cytometry; B - cell growth in the 6 groups was determined by the Cell Counting Kit-8 assay; cells overexpressing ARHGAP1 had the lowest growth rate. All data are presented as mean (SD) from at least 3 independent experiments $(n>3) ; \quad$ a $\quad P<0.01$ vs control with a blank vector (ARHGAP1+NC) or siRNA (ARHGAP1- or ARHGAP1-NC) were between that of normal cells and cells overexpressing ARHGAP1, all showing an apoptotic ratio of nearly $8 \%$ (FIGURE 4A; Supplementary material, Figure S4A). Cells overexpressing ARHGAP1 and cultured under hypoxic conditions showed dramatically reduced cell viability compared with control. In contrast, the cells deficient in ARHGAP1 had the highest growth rates (FIGURE 4B).

Following overexpression of ARHGAP1 (ARHGAP1+) in primary cultured cells and cell lines, both LDH activities increased. Compared with untreated cells (control), LDH release was 4-fold higher in primary myocytes and 7-fold higher in the H9c2 cell line. The maximum LDH activity for cells overexpressing ARHGAP1 was 14 unit/l. Cells transfected with siRNA (ARHGAP1- or ARHGAP1-NC) or blank vectors (ARHGAP1+NC) showed the same results as unprocessed cells: the activity of about 2 unit/l in primary cultured myocytes and of about 2.5 unit/l in cell lines (Supplementary material, Figure S4B and S4C).

Effect of ARHGAP1 overexpression on promitotic or remodeling regulators Considering the role of ARHGAP1 and the phenomena observed under hypoxic conditions, the mRNA expression and protein levels of some tumor-related genes were analyzed by qPCR and Western blot, respectively. The proteins MMP2, CCNB1, and PCNA are all highly involved in cancer cell proliferation and metastasis. When ARHGAP1 was overexpressed, the mRNA expression of MMP2, CCNB1, and PCNA was suppressed (Supplementary material, Figure S5A). In contrast, when ARHGAP1 was sustainedly knocked down, the mRNA expression of MMP2, CCNB1, and PCNA was elevated. At the same time, control cells and those with blank vectors showed identical mean mRNA levels for these proteins. Simultaneously, the levels of MMP2, CCNB1, and PCNA were estimated by Western blot (FIGURE 5). The results were similar to those obtained with qPCR after the protein levels were quantified and normalized to tubulin (Supplementary material, Figure S5B).

Effect of ARHGAP1 overexpression on death modulator genes in $\mathbf{H} 9 \mathrm{c} 2$ cells Ischemic cardiomyopathy is invariably associated with programmed cell death. In this study, we analyzed the apoptosis modulators Bcl-2 and Bax, as well as the crucial cell death effector caspase- 3 . When H9c2 cells overexpressed ARHGAP1, the levels of the antiapoptotic protein Bcl-2 and the proapoptotic protein Bax were suppressed and elevated, respectively (Supplementary material, Figure S6A). Moreover, Western blot revealed that the levels of the apoptosis effector pro-caspase- 3 and those of cleaved caspase- 3 decreased and increased, respectively, when 


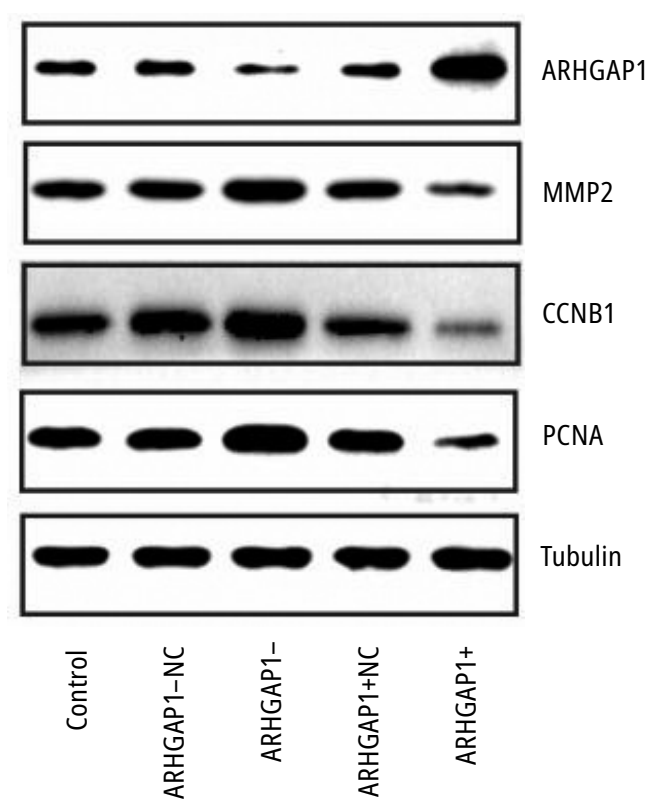

FIGURE 5 Association between cell proliferation effectors and ARHGAP1 overexpression in the myoblast cells: control (untreated H9c2 cells); ARHGAP1- (cells transfected with siRNA targeting ARHGAP1); ARHGAP1-NC (cells transfected with irrelevant siRNA sequence); ARHGAP1+ (cells containing vectors overexpressing ARHGAP1); and ARHGAP1+NC (cells with only blank vectors). The expression levels of ARHGAP1, matrix metallopeptidase 2 (MMP2), cyclin B1 (CCNB1), and proliferating cell nuclear antigen (PCNA) were determined by Western blot.

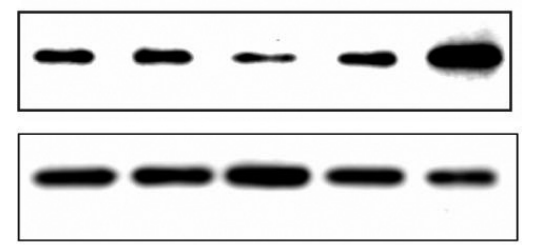

ARHGAP1

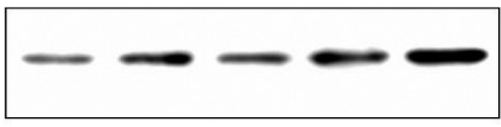

BAX

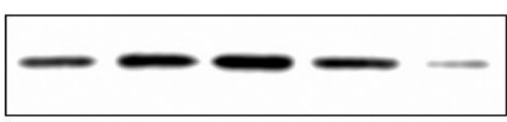

Pro-caspase-3

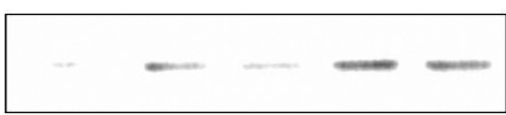

Cleaved caspase-3

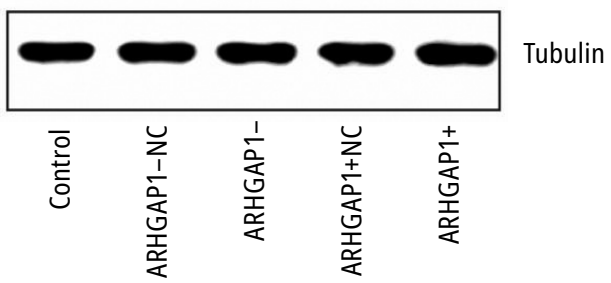

FIGURE 6 Association between cell apoptotic modulators and ARHGAP1 overexpression in the myoblast cells: control (untreated H9c2 cells); ARHGAP1- (cells transfected with siRNA targeting ARHGAP1); ARHGAP1-NC (cells transfected with irrelevant siRNA sequence); ARHGAP1+ (cells containing vectors overexpressing ARHGAP1); and ARHGAP1+NC (cells with only blank vectors). The levels of ARHGAP1, BCl-2, Bax, pro-caspase-3, and cleaved caspase-3 proteins were determined by Western blot.
ARHGAP1 was overexpressed (FIGURE 6; Supplementary material, Figure S6B). With ARHGAP1 deficiency, the opposite was observed: the levels of Bax were suppressed, whereas those of Bcl-2 were elevated. Simultaneously, most of the caspase- 3 adopted the form of pro-caspase-3. Untreated cells and cells transfected with blank vectors showed intermediate levels of $\mathrm{Bcl}-2, \mathrm{Bax}$, pro-caspase-3, and cleaved caspase-3.

DISCUSSION Ischemic cardiomyopathy is the primary cause of more than $60 \%$ of heart failure cases worldwide and is induced by restricted blood supply to the cardiac muscles and the subsequent low levels of oxygen. In ICM, cardiac myocytes undergo a series of remodeling of programmed cell death. ${ }^{2}$ Therefore, therapies specifically targeting apoptosis are expected to improve the prognosis of patients with ischemic heart disease or heart failure and have attracted considerable attention from the scientific community. Currently, most data are ambiguous because different studies have used different models, and no single model can fully mimic the properties of the adult heart. In our study, we attempted to find a novel effector of ICM by setting up cellular and animal models, and to disclose the underlying mechanism of ICM.

ARHGAP1, a member of the Rho GAP family, is widely studied for its involvement in different types of cancer as well as in the different stages of cancer progression. ${ }^{8,17}$ The analysis of the 4 published databases of patients with ICM revealed that the overexpression of ARHGAP1 was a common link between these patients, thus implying the important role of ARHGAP1 in programmed cell death associated with ICM.

To replicate the presence of ARHGAP1 in ICM, we successfully established a myocardial hypoxia model as well as an ICM rat model. Increased ARHGAP1 was detected in both these models. To clarify the mechanism of ARHGAP1 in myocardial cells, the gene was either overexpressed or knocked down in H9c2 cells. All the results supported the hypothesis that ARHGAP1 induced cell apoptosis and suppressed cell proliferation. The underlying pathway of apoptosis was further elucidated by analyzing the regulation of $\mathrm{Bcl}-2 / \mathrm{Bax}$ and pro-caspase-3/ cleaved caspase-3. We showed that ARHGAP1 overexpression results in reduced levels of $\mathrm{Bcl}-2$ and increased levels of Bax. This, in turn, initiates the activity of apoptosis effector caspase-3, and subsequently, cell death. The growth suppression was caused by the downregulation of the tumor-related proteins MMP2, CCNB1, and PCNA, which are all involved in tumor progression and metastasis. ${ }^{18-20}$ Overall, it appears that ARHGAP1 exerts bilateral effects in ICM 
development: it suppresses proliferation and stimulates apoptosis. To the best of our knowledge, this is the first study to describe the function of ARHGAP1 in myocardial cells and to elucidate its potential relationship with ICM.

Previous studies reported ARHGAP1 to have an antitumor effect in different types of cancer $^{21-23}$ as well as to be associated with cell apoptosis during embryogenesis. ${ }^{12}$ On the other hand, in this research, ARHGAP1 was found to be involved in hypoxia-induced apoptosis, and it played a vital role in the transition to programmed cell death. Therefore, as a potentially reversible cause of cardiac function deterioration, ARHGAP1 is expected to become a novel molecular marker and therapy target in patients with ICM.

Ischemic cardiomyopathy is an extremely complex syndrome, characterized by necrosis and apoptosis of myocardial cells. Free radical release, mitochondrial damage, protease activation, and oxidative stress, among other factors, are involved in the development of ICM as well as glucose deprivation cell model. ${ }^{24}$ By using animal and cellular models, ARHGAP1 was shown to be highly correlated with ischemia. However, further research is needed to elucidate its involvement in the complex pathway that leads to the activation of cell apoptosis and inhibition of cell proliferation.

In conclusion, our study was the first to investigate ARHGAP1 overexpression under hypoxic conditions in the cellular model of myocrdial ischemia and the rat model of ICM. Furthermore, the function of ARHGAP1 in inducing apoptosis and suppressing proliferation was validated and the underlying pathway involving regulation of Bcl-2/Bax and pro-caspase-3/cleaved caspase-3 was explored. Through suppressing tumor-related gene of MMP2/CCNB1/PCNA and regulating apoptosis modulators Bcl-2/Bax, ARHGAP1 promoted the hypoxia-induced apoptosis of myocardial cells.

\section{SUPPLEMENTARY MATERIAL}

Supplementary material is available at www.mp.pl/kardiologiapolska.

\section{ARTICLE INFORMATION}

CONTRIBUTION STATEMENT QQ conceived the concept of and supervised the study. XZ designed experiments and wrote the original manuscript. JG established a rat model of ischemic cardiomyopathy. MG and HD cultured cells and performed cell-based assays. SC, CZ, and YW analyzed the data.

CONFLICT OF INTEREST None declared.

OPEN ACCESS This is an Open Access article distributed under the terms of the Creative Commons Attribution-NonCommercial-NoDerivatives 4.0 International License (CC BY-NC-ND 4.0), allowing third parties to download articles and share them with others, provided the original work is properly cited, not changed in any way, distributed under the same license, and used for noncommercial purposes only. For commercial use, please contact the journal office at kardiologiapolska@ptkardio.pl.

HOW TO CITE Zhang X, Guan J, Guo M, et al. Rho GTPase-activating protein 1 promotes apoptosis of myocardial cells in an ischemic cardiomyopathy model. Kardiol Pol. 2019; 77: 1163-1169. doi:10.33963/KP.15040

\section{REFERENCES}

1 Li Y, Du Y, Cao J, et al. MiR-130a inhibition protects rat cardiac myocytes from hypoxia-triggered apoptosis by targeting Smad4. Kardiol Pol. 2018; 76: 993-1001.
2 Osmancik P, Louckova A. Biomarkers of apoptosis, inflammation, and cardiac extracellular matrix remodelling in the prognosis of heart failure. Kardiol Pol. 2017; 75: 295-305.

3 Kong SW, Hu YW, Ho JW, et al. Heart failure-associated changes in RNA splicing of sarcomere genes. Circ Cardiovasc Genet. 2010; 3: 138-146.

4 Gronich N, Kumar A, Zhang Y, et al. Molecular remodeling of ion channels, exchangers and pumps in atrial and ventricular myocytes in ischemic cardiomyopathy. Channels (Austin). 2010; 4: 101-107.

5 Kittleson MM, Minhas KM, Irizarry RA, et al. Gene expression analysis of ischemic and nonischemic cardiomyopathy: shared and distinct genes in the development of heart failure. Physiol Genomics. 2005; 21: 299-307.

6 Etienne-Manneville S, Hall A. Rho GTPases in cell biology. Nature. 2002; 420: 629-635.

7 Haga RB, Ridley AJ. Rho GTPases: regulation and roles in cancer cell biology. Small GTPases. 2016; 7: 207-221.

8 Vega FM, Ridley AJ. Rho GTPases in cancer cell biology. FEBS Lett. 2008; 582 : 2093-2101.

9 Johnstone CN, Castellvi-Bel S, Chang LM, et al. ARHGAP8 is a novel member of the RHOGAP family related to ARHGAP1/CDC42GAP/p50RHOGAP: mutation and expression analyses in colorectal and breast cancers. Gene. 2004; 336: 59-71.

$10 \mathrm{Xu}$ J, Zhou $X$, Wang J, et al. RhoGAPs attenuate cell proliferation by direct interaction with p53 tetramerization domain. Cell Rep. 2013; 3: 1526-1538.

11 Satterfield L, Shuck R, Kurenbekova L, et al. miR-130b directly targets ARHGAP1 to drive activation of a metastatic CDC42-PAK1-AP1 positive feedback loop in Ewing sarcoma. Int J Cancer. 2017; 141: 2062-2075.

12 Wang L, Yang LD, Burns K, et al. Cdc42GAP regulates C-Jun N-terminal kinase (JNK)-mediated apoptosis and cell number during mammalian perinatal growth. Proc Natl Acad Sci U S A. 2005; 102: 13 484-13489.

13 Cook SA, Sugden PH, Clerk A. Regulation of bcl-2 family proteins during development and in response to oxidative stress in cardiac myocytes: association with changes in mitochondrial membrane potential. Circ Res. 1999; 85: 940-949.

14 Hengartner MO. The biochemistry of apoptosis. Nature. 2000; 407: 770-776.

15 Azhar G, Liu L, Zhang X, Wei JY. Influence of age on hypoxia/reoxygenation-induced DNA fragmentation and bcl-2, bcl-xl, bax and fas in the rat heart and brain. Mech Ageing Dev. 1999; 112: 5-25.

16 Ekhterae D, Lin Z, Lundberg MS, et al. ARC inhibits cytochrome c release from mitochondria and protects against hypoxia-induced apoptosis in heart-derived H9c2 cells. Circ Res. 1999; 85: e70-e77.

17 Nagaraja GM, Kandpal RP. Chromosome $13 q 12$ encoded Rho GTPase activating protein suppresses growth of breast carcinoma cells, and yeast two-hybrid screen shows its interaction with several proteins. Biochem Biophys Res Commun. 2004; 313: 654-665.

18 Moroz A, Delella FK, Almeida R, et al. Finasteride inhibits human prostate cancer cell invasion through MMP2 and MMP9 downregulation. PLoS One. 2013; $8: \mathrm{e} 84757$.

19 Mitra A, Chakrabarti J, Banerji A, et al. Culture of human cervical cancer cells, SiHa, in the presence of fibronectin activates MMP-2. J Cancer Res Clini Oncol. 2006; 132: 505-513.

20 Mitra A, Chakrabarti J, Chattopadhyay N, Chatterjee A. Membrane-associated MMP-2 in human cervical cancer. J Environ Pathol Toxicol Oncol. 2003; 22: 93-100. 21 Hankins GR, Sasaki T, Lieu AS, et al. Identification of the deleted in liver cancer 1 gene, Dlc1, as a candidate meningioma tumor suppressor. Neurosurgery. 2008; 63: 771-780.

22 Ching YP, Wong CM, Chan SF, et al. Deleted in liver cancer (DLC) 2 encodes a RhoGAP protein with growth suppressor function and is underexpressed in hepatocellular carcinoma. J Biol Chem. 2003; 278: 10824-10830.

23 Wong CM, Yam JWP, Ching YP, et al. Rho GTPase-activating protein deleted in liver cancer suppresses cell proliferation and invasion in hepatocellular carcinoma. Cancer Res. 2005; 65: 8861-8868.

24 Wei S, Tong J, Xue Q, et al. Pathways involved in oxygen glucose deprivation damage of astrocytes. J Mol Neurosci. 2017; 61: 115-122. 